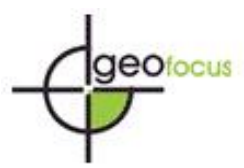

Suárez, M. E., Martínez Fernández, F. H., Pedro Pérez, A. A. y Belmonte Jiménez, S. I. (2020). Simulación del movimiento de partículas contaminantes sobre zonas de vulnerabilidad en el acuífero de los valles centrales de Oaxaca. GeoFocus, Revista Internacional de Ciencia y Tecnología de la Información Geográfica, 25, 3-25. http://dx.doi.org/10.21138/GF.653

\title{
SIMULACIÓN DEL MOVIMIENTO DE PARTÍCULAS CONTAMINANTES SOBRE ZONAS DE VULNERABILIDAD EN EL ACUÍFERO DE LOS VALLES CENTRALES DE OAXACA
}

\author{
MARIO ERNESTO SUÁREZ-MOTA ${ }^{1}$, FERNANDO HUMBERTO MARTINEZ HERNÁNDEZ*1, \\ ALMA ALHELI PEDRO PÉREZ ${ }^{1}$ Y SALVADOR ISIDRO BELMONTE JIMÉNEZ ${ }^{2}$ \\ ${ }^{1}$ Universidad de la Sierra Juárez \\ Av. Universidad s/n, Barrio la Asunción, Ixtlán de Juárez, Oaxaca México. C.P. 68725 \\ ${ }^{2}$ Dirección General, Centro Inter. de Investigación para el Desarrollo Integral Regional, Oaxaca. \\ Hornos No. 1003, Col. Noche Buena, Municipio de Santa Cruz Xoxocotlán, Oaxaca..C.P. 71230. \\ fermartinezher@gmail.com*, mesuarez@unsij.edu.mx
}

\section{RESUMEN}

El presente trabajo integra el uso de dos metodologías de índices y superposición: Matus para definir zonas potenciales de recarga hídrica y DRASTIC para reconocer la vulnerabilidad del acuífero. Además, se hizo una modelación del acuífero de los Valles Centrales de Oaxaca con MODFLOW, aunado a una simulación de partículas con el módulo MODPATH. Se encontró que las zonas más vulnerables a contaminantes son: el área de cultivo localizada a $1.5 \mathrm{~km}$ al noroeste de San Lucas Quiavini, el cuerpo de agua localizado a $2.5 \mathrm{~km}$ al noroeste de San Miguel Tlanichico, terrenos de cultivo entre Guadalupe y San Sebastián Etla y la zona urbana de San Francisco Telixtlahuaca. Estas áreas no solo son un peligro para la calidad de agua en el sitio, sino que podrían afectar a pozos o comunidades a kilómetros de distancia, por lo que requieren especial protección.

Palabras clave: DRASTIC; MODFLOW; MODPATH; vulnerabilidad; acuífero; Oaxaca.

\section{SIMULATION OF MOVEMENT OF POLLUTING PARTICLES IN AREAS OF VULNERABILITY IN THE AQUIFER VALLEYS CENTRALS OF OAXACA}

\section{ABSTRACT}

This study is an approach combined with the use of two indices and overlap methodologies: Matus for potential recharge zones and DRASTIC for aquifer vulnerability. In addition, an aquifer model of the Central Valleys made with MODFLOW software, together with a particle simulation method with the MODPATH package. It was found that the most vulnerable zones are: the cultivation area located $1.5 \mathrm{~km}$ northwest of San Lucas Quiavini, the body of water at $2.5 \mathrm{~km}$ northwest of San Miguel Tlanichico, 


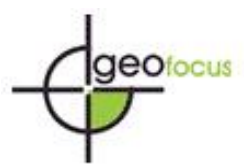

Suárez, M. E. (2020). Simulación del movimiento de partículas contaminantes sobre zonas de vulnerabilidad en el acuífero de los valles centrales de Oaxaca. GeoFocus, Revista Internacional de Ciencia y Tecnología de la Información Geográfica, 25, 3-25. http://dx.doi.org/10.21138/GF.653

agricultural land between Guadalupe and San Sebastián Etla and the urban area of San Francisco Telixtlahuaca. These areas are not only a danger for the water quality in the site, but can also affect nearby wells or distant communities, so that require special protection.

Keywords: DRASTIC, MODFLOW, MODPATH, vulnerability, aquifer, Oaxaca.

\section{Introducción}

El agua es fundamental para la vida en la Tierra, pero solo una mínima parte de ella es apta para el consumo humano y las principales reservas son aguas subterráneas (Bokova 2010). En México, se tienen pocos estudios a escala nacional y local de la contaminación de los acuíferos y las zonas susceptibles a la degradación de la calidad de las aguas subterráneas (Foster e Hirata 1991, Becerril, 2013).

En el caso particular del acuífero de los Valles Centrales que abastece a la ciudad de Oaxaca y a 20 de sus municipios conurbados tiene serios problemas de contaminación (Velásquez 2017). Este es un conflicto muy grave de salud pública y de abastecimiento ya que la calidad y cantidad de agua de este acuífero se está deteriorando alarmantemente (Bautista-Perdomo 2013). Por ello, es necesario estudiar la vulnerabilidad de este acuífero ante riesgos de contaminación, para poder contar con herramientas que sean útiles para la conservación, gestión y protección del recurso hídrico.

En el contexto hidrogeológico, la vulnerabilidad es la posibilidad de infiltración y difusión de un contaminante en un acuífero (Albinet y Margat 1970). Esta puede ser intrínseca o específica, la primera es independiente de la naturaleza del contaminante, mientras que la otra se utiliza para definir la vulnerabilidad del agua subterránea asociada con algún contaminante en particular o, con un grupo de contaminantes (Gogu y Dessargues 2000, Becerril 2013). Existen cuatro tipos de métodos para evaluar la vulnerabilidad a la contaminación: modelos de simulación, métodos estadísticos, de índices y superposición y los de ambientes hidrogeológicos (Vargas y Tosse 2010). La computadora ha sido un progreso considerable en la evaluación de estos métodos, como manera económica y rápida en el estudio del movimiento, el equilibrio y gestión operacional de los acuíferos (Koohestani et al. 2013).

Los sistemas de información geográfica (SIG) permiten el análisis de la vulnerabilidad que tienen los acuíferos a contaminantes a través de métodos de superposición de mapas que utilizan índices que evalúan la profundidad, recarga neta, tipo de acuífero, suelo, topografía, conductividad y zona saturada (DRASTIC, por sus siglas en inglés; Esteller et al. 2002). También el uso de modelos matemáticos con la solución numérica se ha convertido en un enfoque apropiado en el estudio del agua subterránea. El software MODFLOW/MODPATH modela sistemas subterráneos y simula el movimiento de partículas. Estos métodos se han utilizado en todo el mundo y su aplicación ha sido comprobada de manera exitosa (Koohestani et al. 2013).

\section{Revisión bibliográfica}

\subsection{Potencial de recarga hídrica}

En términos generales, se denomina recarga hídrica al proceso por el cual se incorpora a un acuífero el agua procedente de fuera del contorno que lo limita (Custodio 1998, Matus et al. 2009). El área o zona 


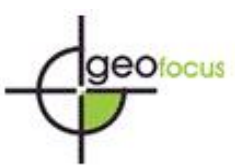

Suárez, M. E. (2020). Simulación del movimiento de partículas contaminantes sobre zonas de vulnerabilidad en el acuífero de los valles centrales de Oaxaca. GeoFocus, Revista Internacional de Ciencia y Tecnología de la Información Geográfica, 25, 3-25. http://dx.doi.org/10.21138/GF.653

donde ocurre la recarga se llama zona de recarga y son sitios donde la capacidad de infiltración es alta (Matus et al. 2009). La metodología propuesta por Matus et al. (2009) identifica las zonas potenciales de recarga hídrica en subcuencas hidrográficas. Esta propuesta se basa principalmente en cinco diferentes requerimientos naturales; el suelo que impide o facilita la infiltración, la topografía que determina el tiempo de contacto entre la superficie y el agua, la geología que afecta grandemente a la recarga hídrica, la cobertura vegetal que disminuye escorrentía e intercepta la lluvia y el uso de suelo que es el elemento más cambiante y con mayor influencia de la actividad humana.

\subsection{Metodología DRASTIC para vulnerabilidad de acuíferos}

El método DRASTIC fue diseñado para evaluar el potencial relativo de contaminación del agua subterránea con conocimiento básico y de fácil uso, aunque no es un sustituto de las inspecciones en el sitio, sino un complemento (Becerril 2013) y se utilizan para la evaluación de la vulnerabilidad siete parámetros; P: Profundidad del agua subterránea, R: Recarga neta, A: Litología y estructura del medio acuífero, S: Tipo de suelo, T: Topografía, I: Naturaleza de la zona no saturada, C: Conductividad hidráulica del acuífero.

\subsection{Modelación del acuífero y simulación de partículas}

El software usado en la modelación tridimensional del acuífero de los Valles Centrales de Oaxaca, fue MODFLOW de ModelMuse. Es un método de diferencias finitas que consiste en una aproximación de las derivadas parciales con expresiones algebraicas de los valores de la variable dependiente en un limitado número de puntos seleccionados (Pollock 2012).

Para la simulación de partículas se utilizó el módulo MODPATH, implementado en MODFLOW, así, se genera un modelo de líneas a partir del flujo de aguas subterráneas en el que se pueden visualizar tres dimensiones, el desplazamiento de las partículas representadas desde una o varias fuentes de origen en un solo periodo o en tiempos parciales de evaluación (Cavero 2013). Este paquete trabaja bajo los supuestos que menciona Pollock (2012):

- El contaminante se infiltró a la zona saturada

- La simulación comienza a partir de que la partícula está en el nivel freático

- El contaminante posee las mismas propiedades que el agua

\section{Materiales, datos y métodos}

En el presente análisis se plantea un enfoque combinado de dos métodos paramétricos, uno de modelación numérica y de simulación de transporte advectivo y otro de sobreposicionamiento. Se determinaron las principales zonas de vulnerabilidad a contaminantes del acuífero de los Valles Centrales con el uso de DRASTIC que se combinó con la metodología de Matus para zonas potenciales de recarga. Además, se realizó un modelo de flujo de aguas subterráneas con el software MODFLOW de ModelMuse para comprender la dinámica del sistema acuífero e identificar la dirección y velocidad de los flujos subterráneos para posteriormente realizar una simulación de partículas en dicho modelo con el módulo MODPATH sobre las zonas con mayor vulnerabilidad. 


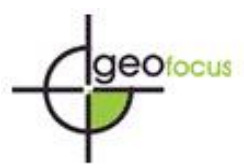

Suárez, M. E. (2020). Simulación del movimiento de partículas contaminantes sobre zonas de vulnerabilidad en el acuífero de los valles centrales de Oaxaca. GeoFocus, Revista Internacional de Ciencia y Tecnología de la Información Geográfica, 25, 3-25. http://dx.doi.org/10.21138/GF.653

\subsection{Delimitación espacial}

El acuífero de los Valles Centrales también llamado Subcuenca Alto Atoyac se ubica en el centro del estado de Oaxaca al sur de México y está formado por tres zonas principales: Valle de Etla, Valle de Zimatlán y Valle de Tlacolula. El acuífero se identifica con la clave OAX25 y 2025 del Sistema de Información Geográfica para el Manejo de las Aguas Subterráneas (SIGMAS) y tiene una superficie aproximada de $3,769 \mathrm{~km}^{2}$ (CONAGUA 2013, figura 1).

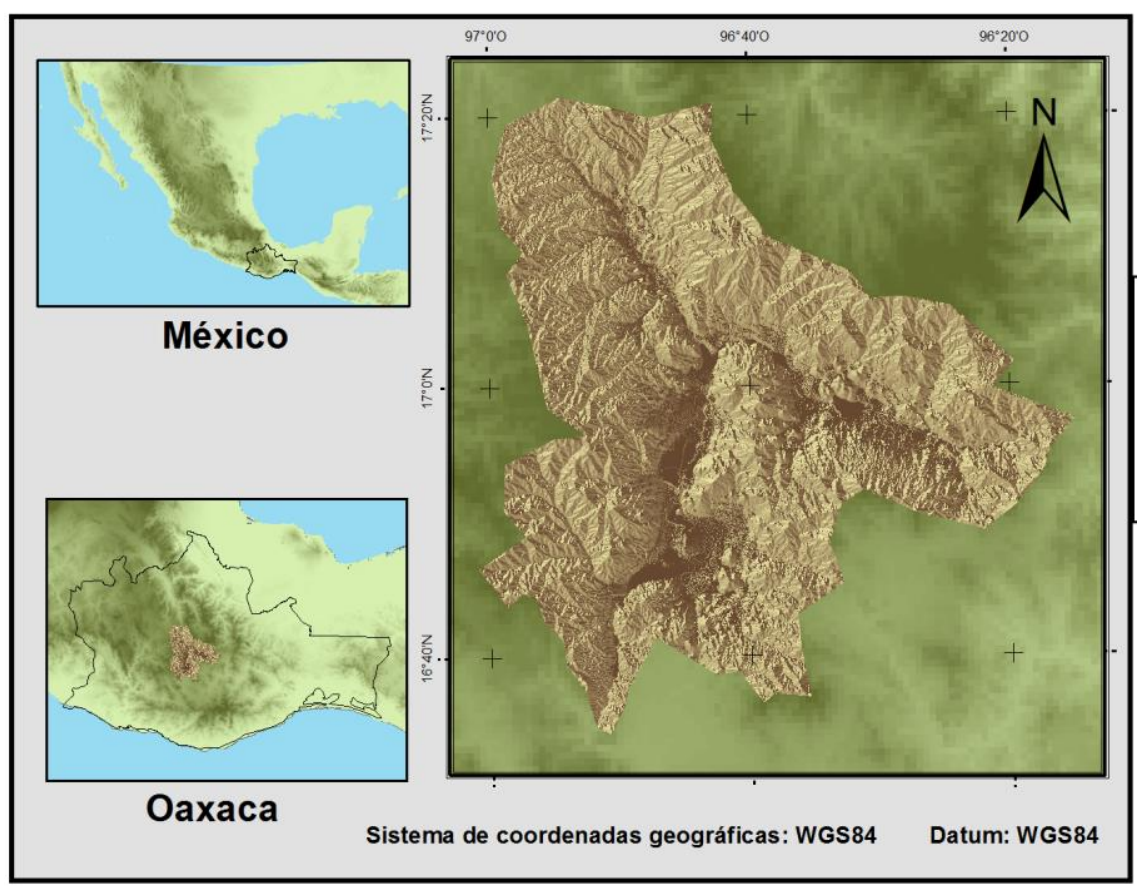

Figura 1.- Localización del acuífero de los Valles Centrales de Oaxaca

\subsection{Caracterización de la subcuenca}

En la subcuenca predomina un clima semiárido cálido con una precipitación promedio entre 450.734 y 935.567 milímetros anuales. Posee 22 usos de suelo y vegetación destacando la agricultura de temporal y la agricultura de riego en la zona aluvial. El tipo de suelo regosol éutrico y la roca gneis son los que más se presentan en la subcuenca. Dentro de la hidrología de la subcuenca se encuentran dos ríos principales; el rio Atoyac, que comienza en el valle de Etla terminando en el Valle de Zimatlán y el rio Salado cuyo caudal comienza en el valle de Tlacolula e intercepta al rio Atoyac en el centro de la subcuenca (CONAGUA 2013, figura 2). 


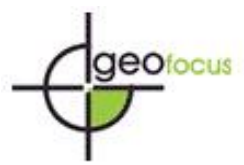

Suárez, M. E. (2020). Simulación del movimiento de partículas contaminantes sobre zonas de vulnerabilidad en el acuífero de los valles centrales de Oaxaca. GeoFocus, Revista Internacional de Ciencia y Tecnología de la Información Geográfica, 25, 3-25. http://dx.doi.org/10.21138/GF.653

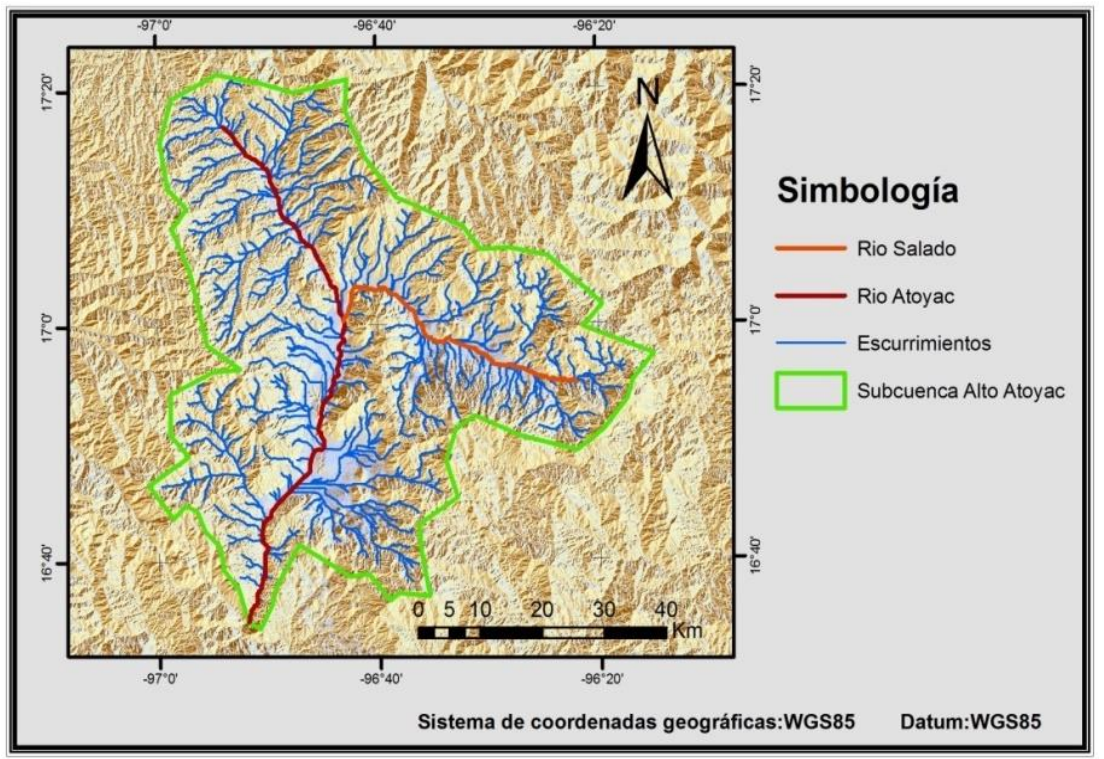

Figura 2. Ríos y escurrimientos presentes en el acuífero.

\subsection{Características del aluvial de los Valles Centrales de Oaxaca}

El área de estudio a modelar es solo la superficie de origen aluvial de tipo libre con una extensión de $1134.65 \mathrm{~km}^{2}$ (figura 3). Está constituido por sedimentos no consolidados como cantos rodados, gravas, arenas, arcillas, y limos formando una mezcla heterogénea (Bautista-Perdomo 2013). El espesor del acuífero varía entre los $10 \mathrm{~m}$ al pie de las Sierras y $200 \mathrm{~m}$ en las partes centrales incluida la zona del Aeropuerto de la ciudad de Oaxaca, el Valle de Etla y Tlacolula (CONAGUA, 2013).

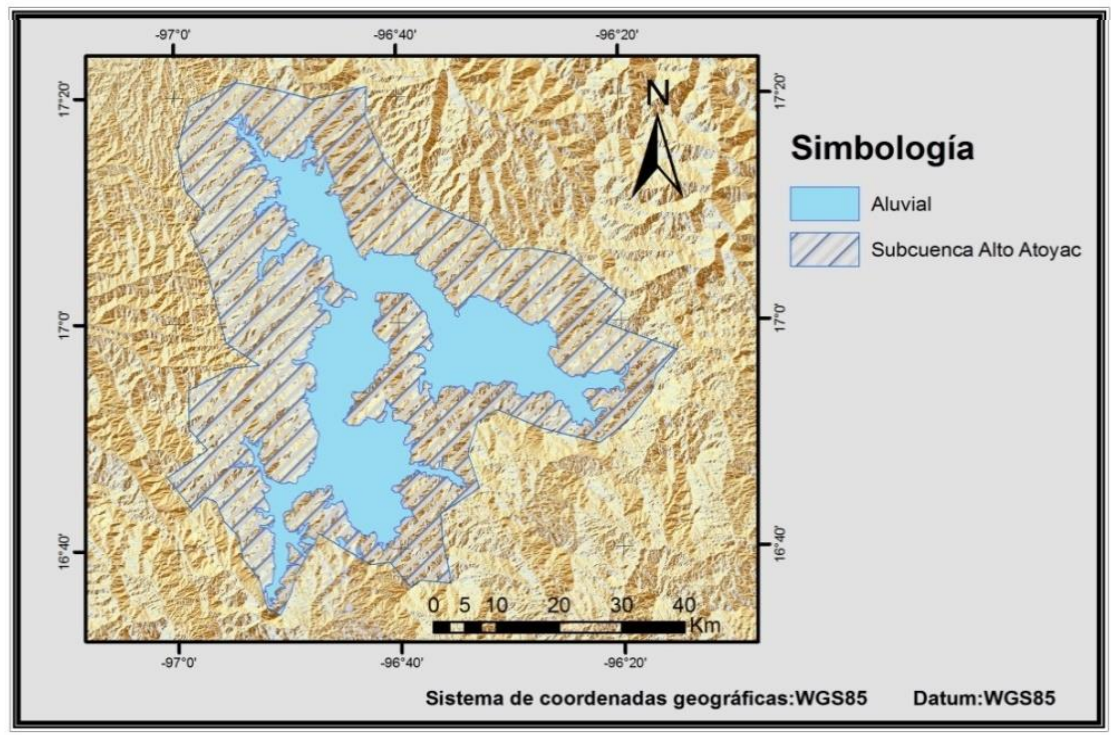

Figura 3. Localización de la superficie aluvial dentro de la Subcuenca Alto Atoyac. 


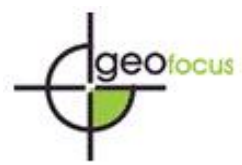

Suárez, M. E. (2020). Simulación del movimiento de partículas contaminantes sobre zonas de vulnerabilidad en el acuífero de los valles centrales de Oaxaca. GeoFocus, Revista Internacional de Ciencia y Tecnología de la Información Geográfica, 25, 3-25. http://dx.doi.org/10.21138/GF.653

La superficie total del acuífero se dividió en celdas de $500 \mathrm{~m}$ por lado $\left(0.25 \mathrm{~km}^{2}\right.$ cada una); se seleccionaron las celdas de la zona aluvial como activas y el resto como celdas inactivas.

Los valores más altos de la conductividad hidráulica se localizan en la zona del aluvial del acuífero desde 5.5 hasta 45 metros por día. Los valores más bajos de 0.05 metros por día se localizan al noroeste de la subcuenca (Bautista-Perdomo, 2013). Los valores más bajos del nivel estático se localizan en los valles de Etla y Zimatlán, aunque hay altas variaciones por los abatimientos generados por la sobreexplotación de los pozos en el acuífero. La recarga estimada del acuífero es de valores cercanos de entre 31 y 165 milímetros anuales (UACh-Unidad Gestora de Servicios Tecnológicos, 2010).

\subsubsection{Zonas potenciales de recarga}

Para la obtención del mapa de las zonas potenciales de recarga se empleó la metodología propuesta por Matus et al. (2009), la cual evalúa cinco elementos biofísicos: Pendiente, obtenida del modelo digital de elevación del Instituto Nacional de Estadística Geografía e Informática (INEGI, 2001), Uso de suelo de INEGI (2014), Cobertura vegetal permanente tomado del estudio mundial de Mao-Ning y Jetz (2014), Geología, obtenido del conjunto de datos vectoriales geológicos de INEGI (2005) y Edafología (INIFAP, 2001). Para ello, se empleó el sistema de información geográfica ArcMap 9.3. La ponderación a usar en la evaluación de cada elemento va de 1 a 5 , donde 1 corresponde al valor más bajo (características menos favorables para que ocurra la recarga hídrica) y 5 , zonas con un potencial muy alto de recarga.

A través del procesamiento de la información digital, se crearon imágenes tipo ráster para cada uno de los parámetros mencionados, a resolución de 15 metros tomando como base el modelo digital de elevación. De igual forma todos los mapas poseen la delimitación del acuífero hecha por la Comisión Nacional del Agua (CONAGUA, 2013).

\subsubsection{DRASTIC}

El método DRASTIC utiliza para la evaluación de la vulnerabilidad siete parámetros. La recarga y el nivel piezométrico se obtuvieron del estudio justificativo del acuífero de los Valles Centrales de Oaxaca (CONAGUA, 2013). Para la conductividad hidráulica se digitalizó un mapa obtenido del estudio de Bautista-Perdomo (2013). El tipo de acuífero y la zona saturada fueron tomados de información cartográfica de INEGI (2005) y la fuente del tipo de suelo y la pendiente se detalla en el apartado 3.3.1. Se usó ArcGIS 9.3 para procesar la información cartográfica y así identificar las zonas de vulnerabilidad. El periodo de tiempo para la evaluación de DRASTIC fue del 2001 al 2009 ya que se encontró toda la información necesaria para este lapso de tiempo.

\subsubsection{Modelación y simulación}

Para la modelación del acuífero se utilizó información de las características del aluvial, como su profundidad, porosidad, estructura y sus capas, esta fue tomada de CONAGUA (2013) y UACh-Unidad Gestora de Servicios Tecnológicos (2010). Además, se utilizó información de los parámetros hidrogeológicos de los índices DRASTIC y Matus.

En la simulación de partículas se utilizaron las zonas de vulnerabilidad obtenidas de la combinación de la metodología Matus y la metodología DRASTIC. Las partículas se simularon en las zonas más vulnerables, a la profundidad del nivel estático. Se obtuvieron seis simulaciones de 1, 10, 25, 50, 75 y 100 años. 


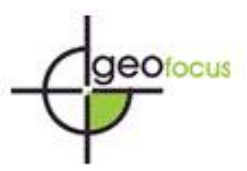

Suárez, M. E. (2020). Simulación del movimiento de partículas contaminantes sobre zonas de vulnerabilidad en el acuífero de los valles centrales de Oaxaca. GeoFocus, Revista Internacional de Ciencia y Tecnología de la Información Geográfica, 25, 3-25. http://dx.doi.org/10.21138/GF.653

\subsection{Técnicas de análisis.}

\subsubsection{Determinación del potencial de recarga}

Para determinar el potencial de recarga hídrica Matus et al. (2009) proponen aplicar la ecuación 1, en la que se sustituye cada parámetro por los valores obtenidos en los análisis.

$$
Z R=[0.27(P e n d)+0.23(T s)+0.12(T r)+0.25(C v e)+0.13(U s)] \quad \text { Ecuación } 1
$$

Siendo Pend: Pendiente, Ts: Tipo de suelo, Tr: Tipo de roca, Cve: Cobertura vegetal permanente, Us: Usos del suelo.

Se analizaron los mapas con las ponderaciones mencionadas anteriormente, se usó la herramienta algebra de mapas implementada en ArcMap 9.3 para resolver la ecuación 1. El grado de recarga se obtuvo a partir de los valores del posible potencial de recarga en el acuífero (Tabla 1).

Tabla 1. Potencial de recarga hídrica según el modelo propuesto.

\begin{tabular}{|c|c|}
\hline Posibilidad de recarga & Rango \\
\hline Muy alta & $4.1-5$ \\
\hline Alta & $3.5-4.9$ \\
\hline Moderada & $2.6-3.49$ \\
\hline Baja & $2-2.59$ \\
\hline Muy baja & $1-1.99$ \\
\hline
\end{tabular}

Fte: Matus et al. 2009.

\subsubsection{Grado de vulnerabilidad con el método DRASTIC}

Una vez asignada la ponderación para cada variable (Tabla 2), el índice de vulnerabilidad se estima con el producto obtenido de la suma de los valores de cada parámetro con su respectivo factor de ponderación:

$$
i V D R A S T I C=(D r \times D w)+(R r \times R w)+(A r \times A w)+(S r \times S w)+(I r \times I w)+(C r+C w) \quad \text { Ecuación } 2
$$

Siendo $i V$ : índice de vulnerabilidad, $r$ : factor de clasificación o ponderación, $w$ : factor de peso. 


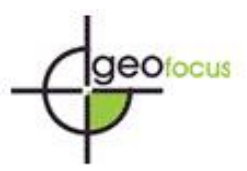

Suárez, M. E. (2020). Simulación del movimiento de partículas contaminantes sobre zonas de vulnerabilidad en el acuífero de los valles centrales de Oaxaca. GeoFocus, Revista Internacional de Ciencia y Tecnología de la Información Geográfica, 25, 3-25. http://dx.doi.org/10.21138/GF.653

Tabla 2. Peso o valoración de cada variable del método DRASTIC.

\begin{tabular}{|c|c|c|c|c|c|c|c|}
\hline Tipo de Variable & $\mathrm{D}_{\mathrm{w}}$ & $\mathrm{R}_{\mathrm{w}}$ & $\mathrm{A}_{\mathrm{w}}$ & $\mathrm{S}_{\mathrm{w}}$ & $\mathrm{T}_{\mathrm{w}}$ & $\mathrm{I}_{\mathrm{w}}$ & $\mathrm{C}_{\mathrm{w}}$ \\
\hline Valoración & 5 & 4 & 3 & 2 & 1 & 5 & 3 \\
\hline
\end{tabular}

Fte. Modificado de Vargas y Tosse 2010

Una vez obtenida la valoración de cada variable, se estima el grado de vulnerabilidad de cada zona en el acuífero de los Valles centrales empleando los rangos correspondientes a los resultados que se obtengan (tabla 3 ).

Tabla 3. Rangos de Vulnerabilidad del método DRASTIC.

\begin{tabular}{|c|c|}
\hline Grado & Vulnerabilidad \\
\hline Muy bajo & $23-64$ \\
\hline Bajo & $65-105$ \\
\hline Moderado & $106-146$ \\
\hline Alto & $147-187$ \\
\hline Muy alto & $188-230$ \\
\hline
\end{tabular}

Fte. Vargas y Tosse 2010.

\subsubsection{Modelación del acuífero en 3D}

Harbaugh (2005) señala la ecuación diferencial parcial que describe los flujos de agua subterránea a través de la porosidad de un acuífero modelado en tres dimensiones (ecuación 3):

$$
\frac{\partial}{\partial x}\left[K x x \frac{\partial h}{\partial x}\right]+\frac{\partial}{\partial y}\left[K y y \frac{\partial h}{\partial y}\right]+\frac{\partial}{\partial z}\left[K z z \frac{\partial h}{\partial z}\right]+W(x, y, z)=S_{s} \frac{\partial h}{\partial t} \quad \text { Ecuación } 3
$$

Siendo $h=$ Carga hidráulica, $t=$ Tiempo, $K_{x x}, K_{y y}, K_{z z}=$ Conductividad hidráulica en las direcciones de los ejes de las coordenadas, $S_{\mathrm{s}}=$ Coeficiente de almacenamiento específico, $W=$ Término general de fuentes y sumideros que define las entradas y salidas del sistema en unidades de volumen por unidad de tiempo. 


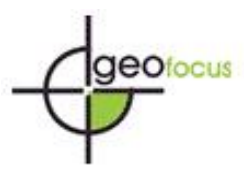

Suárez, M. E. (2020). Simulación del movimiento de partículas contaminantes sobre zonas de vulnerabilidad en el acuífero de los valles centrales de Oaxaca. GeoFocus, Revista Internacional de Ciencia y Tecnología de la Información Geográfica, 25, 3-25. http://dx.doi.org/10.21138/GF.653

\section{Descripción y análisis de resultados}

\subsection{Zonas potenciales de recarga}

El mapa de las zonas potenciales de recarga señala que predominan las zonas con un potencial de recarga moderado, seguido del potencial alto y muy alto (figura 4). Los potenciales de recarga altos y muy altos se ubican principalmente en el sur del valle de Tlacolula y en todo el valle de Etla, teniendo también una gran influencia en zonas cercanas al aeropuerto de la ciudad de Oaxaca y localidades como Santa Cruz Xoxocotlán, Cuilápam de Guerrero, Villa de Zaachila y San Raymundo Jalpam. Las zonas con baja y muy baja recarga están principalmente en el centro de la ciudad de Oaxaca, al norte del valle de Tlacolula y al este del valle de Zimatlán.

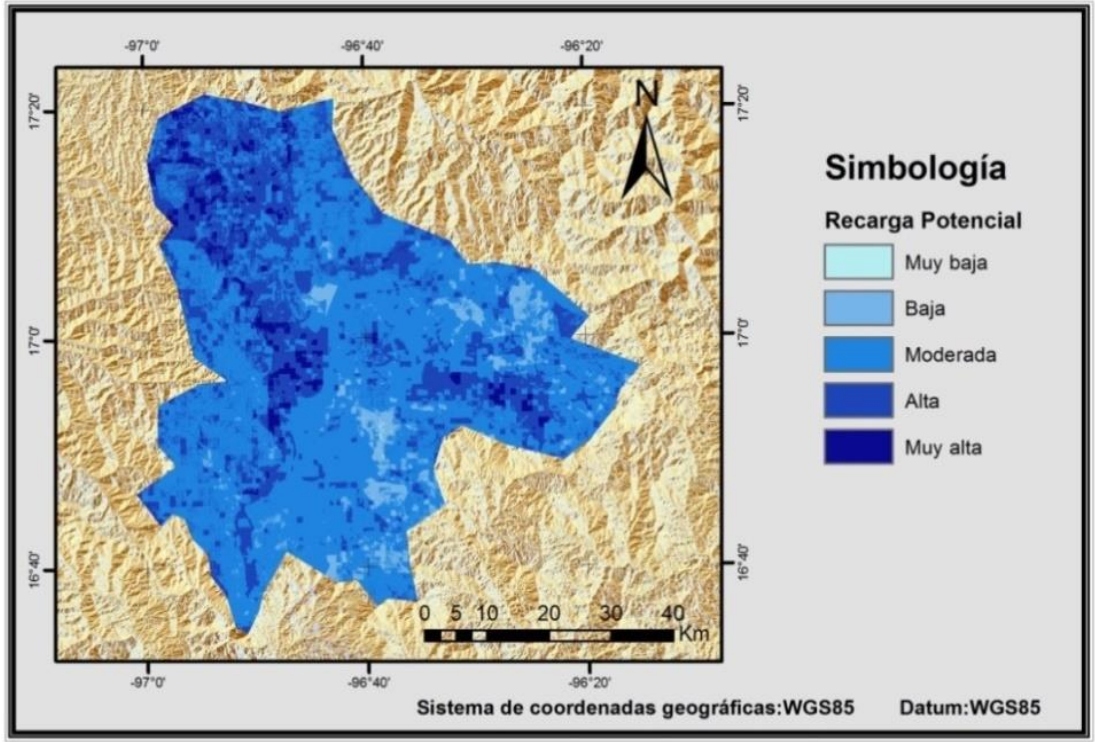

Figura 4. Zonas potenciales de recarga hídrica en la subcuenca Alto Atoyac.

\subsection{Zonas de vulnerabilidad a contaminantes}

El acuífero de los Valles Centrales de Oaxaca presenta tres grados de vulnerabilidad; baja, moderada y alta (figura 5). Las zonas con vulnerabilidad baja representan el $32.58 \%$ del área de la subcuenca $\left(1227.94 \mathrm{~km}^{2}\right)$, las zonas con vulnerabilidad moderada representan el $58.05 \%\left(2188.24 \mathrm{~km}^{2}\right) \mathrm{y}$ las zonas con vulnerabilidad alta ocupan el $9.35 \%$ de la subcuenca $\left(352.40 \mathrm{~km}^{2}\right)$. Las zonas con vulnerabilidad moderada se distribuyen por todo el acuífero ocupando más de la mitad de este. Las zonas con vulnerabilidad alta se localizan principalmente en el aluvial en los valles de Etla y Tlacolula y una pequeña parte al sur de la subcuenca. 


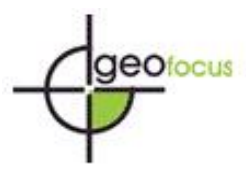

Suárez, M. E. (2020). Simulación del movimiento de partículas contaminantes sobre zonas de vulnerabilidad en el acuífero de los valles centrales de Oaxaca. GeoFocus, Revista Internacional de Ciencia y Tecnología de la Información Geográfica, 25, 3-25. http://dx.doi.org/10.21138/GF.653

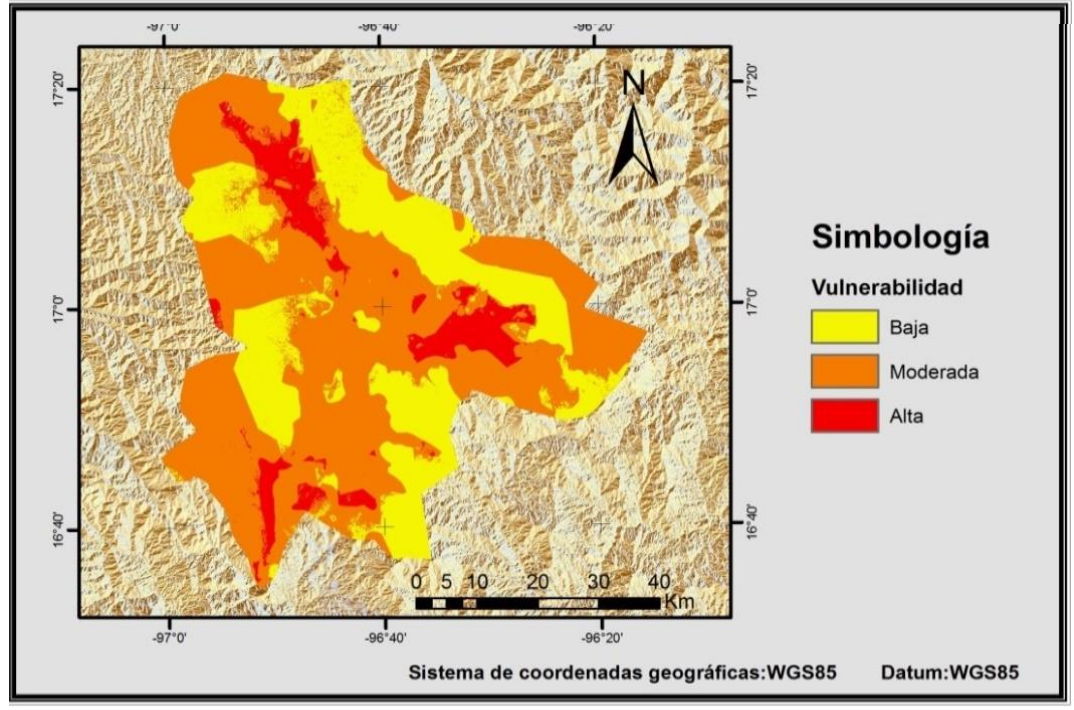

Figura 5. Zonas de vulnerabilidad a contaminantes.

\subsection{Relación entre las Zonas Potenciales de Recarga y el método DRASTIC}

Se muestran en rojo áreas con los valores más altos de las metodologías combinadas, representa la suma de las zonas con alta vulnerabilidad con ponderación 4 y las zonas con muy alta recarga potencial con ponderación 5 dando un resultado de 9 . Estas áreas se localizan específicamente en los valles de Etla y Tlacolula, no obstante, pequeñas zonas no visibles se encuentran al sur de la subcuenca y representan únicamente el $0.37 \%$ de la superficie total.

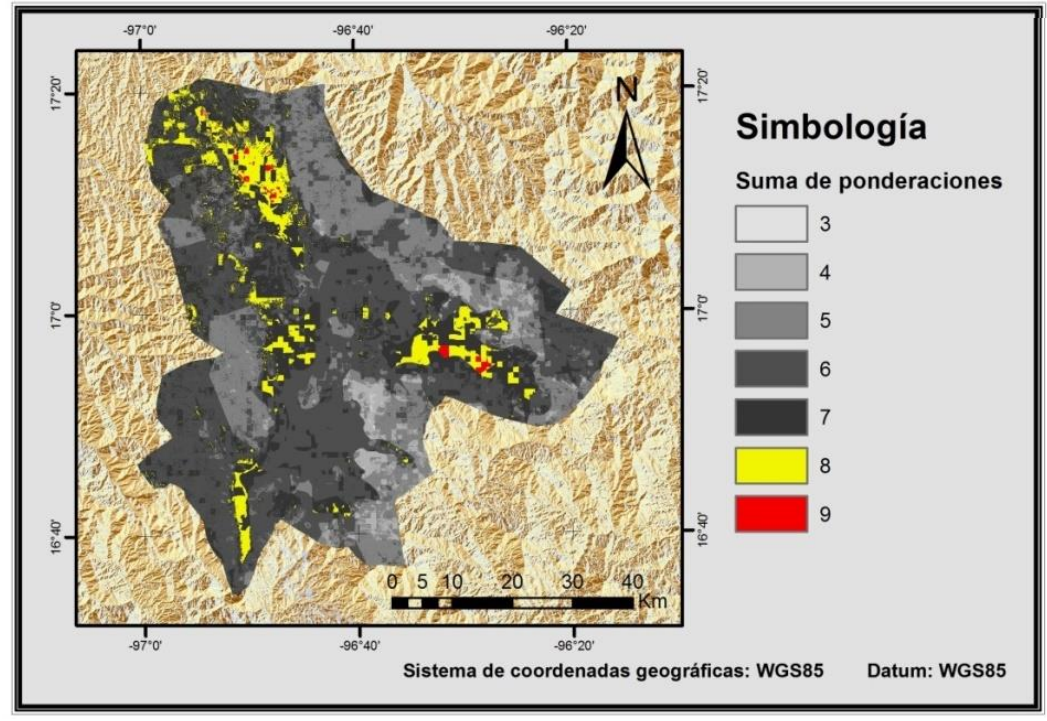

Figura 6. Combinación de las metodologías DRASTIC y zonas potenciales de recarga. 


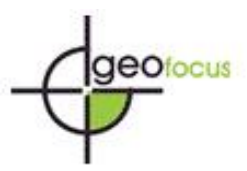

Suárez, M. E. (2020). Simulación del movimiento de partículas contaminantes sobre zonas de vulnerabilidad en el acuífero de los valles centrales de Oaxaca. GeoFocus, Revista Internacional de Ciencia y Tecnología de la Información Geográfica, 25, 3-25. http://dx.doi.org/10.21138/GF.653

Las zonas en amarillo muestran dos intersecciones; la suma de las zonas con vulnerabilidad alta con ponderación 4 y alto potencial de recarga con ponderación 4 y la suma de zonas con vulnerabilidad moderada con ponderación 3 y de muy alta recarga con ponderación 5 cualquiera de ellas dando un resultado de 8 . Dichas zonas se distribuyen principalmente a lo largo del aluvial abarcando el $6.77 \%$ de la superficie del acuífero (figura 6). Se realizó una correlación de Pearson y se obtuvo un resultado de $\mathrm{r}^{2}=0.162$, existiendo una baja correlación entre las zonas potenciales de recarga y de vulnerabilidad.

\subsection{Modelado del acuífero en tercera dimensión}

Con los resultados obtenidos se observa que los flujos subterráneos de la zona de Tlacolula van al noroeste y los de Etla al sureste, ambos yendo hacia la ciudad de Oaxaca. En la parte del Aeropuerto, Zaachila y el valle de Zimatlán, los flujos son al sur, mientras que, en Ocotlán, las aguas subterráneas se mueven hacia el oeste (figura 7).

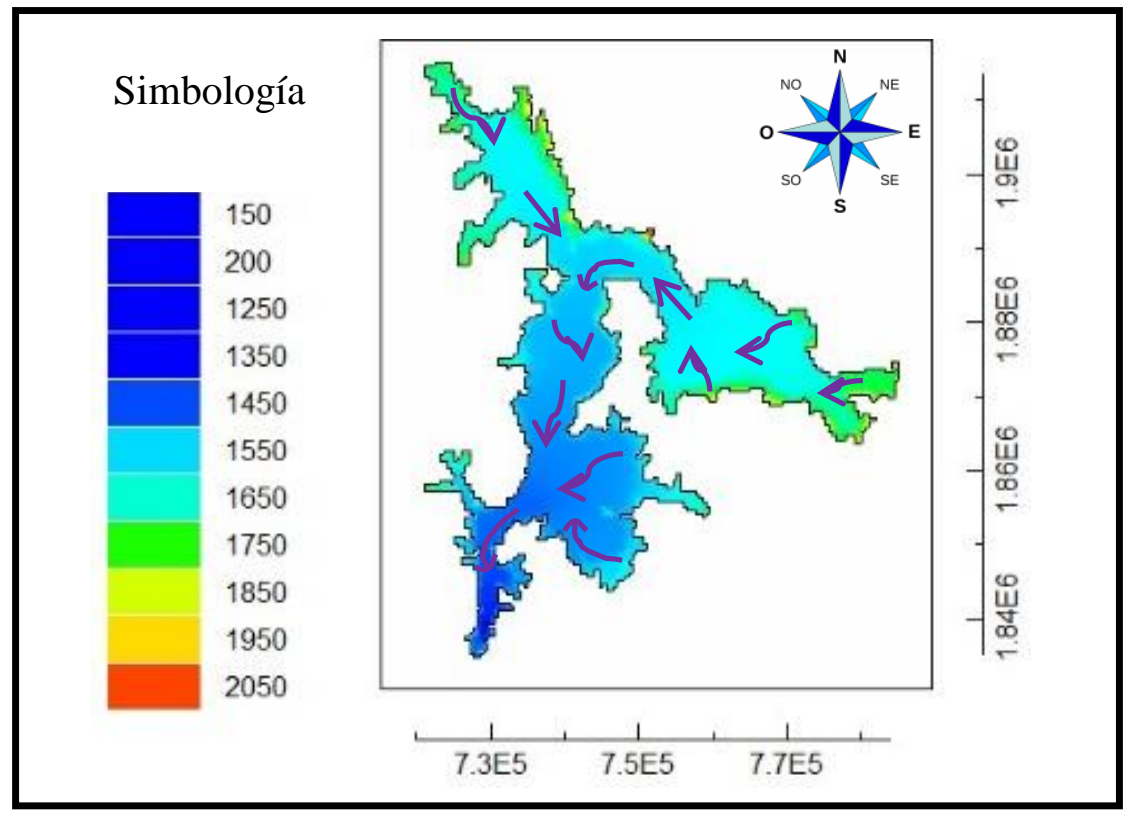

Figura 7. Direcciones de flujo del acuífero de los Valles Centrales.

\subsection{Simulación de partículas contaminantes}

Con el módulo MODPATH de MODFLOW, se simularon seis eventos de contaminación con un campo de partículas para observar su trayectoria y tiempo de viaje. Las partículas comenzaron la simulación sobre las zonas de vulnerabilidad con su respectiva profundidad en el nivel piezométrico. Las zonas vulnerables del centro y el sur del aluvial se ampliaron para su observación. 


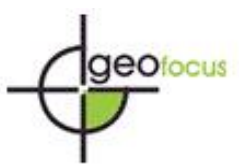

Suárez, M. E. (2020). Simulación del movimiento de partículas contaminantes sobre zonas de vulnerabilidad en el acuífero de los valles centrales de Oaxaca. GeoFocus, Revista Internacional de Ciencia y Tecnología de la Información Geográfica, 25, 3-25. http://dx.doi.org/10.21138/GF.653

En el año uno de la simulación, en el valle de Tlacolula se observó un recorrido en una zona vulnerable a $1.5 \mathrm{~km}$ al noroeste de San Lucas Quiavini donde se registró un desplazamiento de partícula de $380 \mathrm{~m}$. A $4 \mathrm{~km}$ de la Cienega Zimatlán las partículas se movieron 250 metros desde su punto inicial. Se observaron desplazamientos en todas las zonas vulnerables del valle de Etla especialmente a $1 \mathrm{~km}$ al norte donde se tiene el mayor desplazamiento a un año con $1325 \mathrm{~m}$ (figura 8A).

En la simulación de 10 años, los mayores desplazamientos en el valle de Tlacolula fueron de 1500 m, mientras que en San Francisco Telixtlahuaca las partículas se movieron $3000 \mathrm{~m} .6 \mathrm{~km}$ al oeste de la Villa de Zaachila se encontró el más largo desplazamiento de esta simulación con 3300 m (figura 8B).

En 25 años los valores en el desplazamiento para Tlacolula van de 1500 a 3000 m, permaneciendo en los valores más altos las partículas cercanas a San Lucas Quiavini. Comienza a decrecer la velocidad de desplazamiento de las partículas de San Francisco Telixtlahuaca con una longitud de $4700 \mathrm{~m}$ llegando a territorio de San Pablo Huitzo. Las partículas del oeste de la villa de Zaachila recorren 3900 m, mientras que en las zonas vulnerables del sur del aluvial comienza el recorrido de las partículas con $1200 \mathrm{~m}$ (figura $8 \mathrm{C})$.

La simulación a 50 años muestra un decaimiento en todos los desplazamientos. En el valle de Tlacolula las partículas recorren entre 3200 y $5300 \mathrm{~m}$ hacia el noroeste respetando la dirección de flujo. En el norte del valle de Etla las partículas se desplazaron un máximo de $5300 \mathrm{~m}$ hacia el sureste. En Zaachila se desplazaron 3950 metros llegando al subsuelo de la villa de Zaachila. En la zona vulnerable al sur del aluvial se visualiza un desplazamiento de 2390 metros siendo este el menor deslizamiento registrado (figura $8 \mathrm{D}$ ).

En las últimas simulaciones de 75 y 100 años se aprecia una partícula en la zona que se encuentra entre Guadalupe y San Sebastián Etla que recorre $13.8 \mathrm{~km}$ y llega hasta la ciudad de Oaxaca. Sin embargo, las partículas que tienen mayor desplazamiento en la simulación son las de Tlacolula con un máximo de 17500 metros. Las partículas del valle de Zaachila, Zimatlán y el sur del aluvial casi no cambiaron su posición quedando en menos de 4000 metros de recorrido (figura 9A y 9B). 


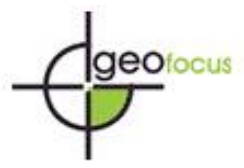

Suárez, M. E. (2020). Simulación del movimiento de partículas contaminantes sobre zonas de vulnerabilidad en el acuífero de los valles centrales de Oaxaca. GeoFocus, Revista Internacional de Ciencia y Tecnología de la Información Geográfica, 25, 3-25. http://dx.doi.org/10.21138/GF.653

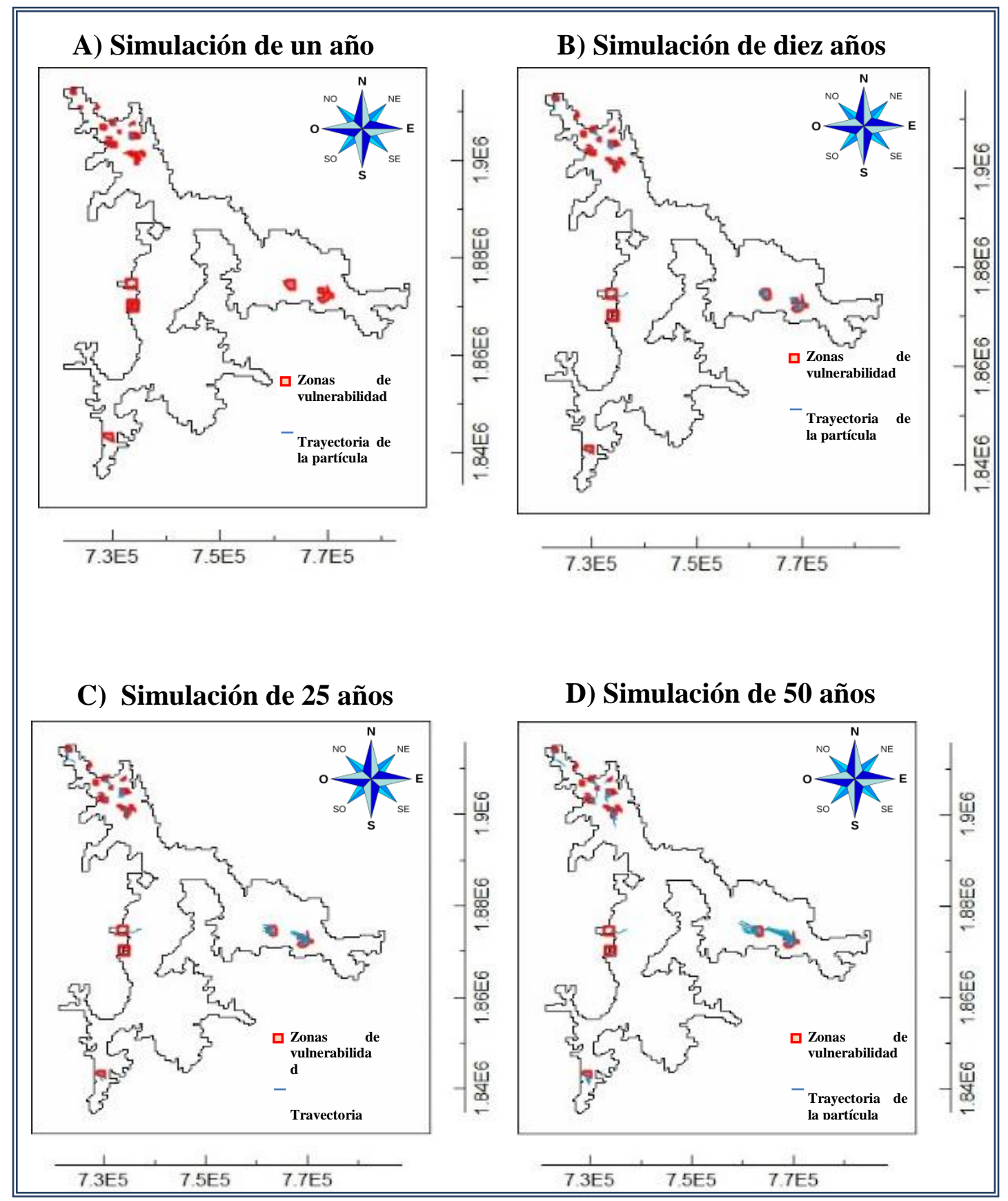

Figura 8. Simulaciones del movimiento de partículas a 1 (A), 10(B), 25(C) y 50 (D) años sobre las zonas de vulnerabilidad en el acuífero de los Valles Centrales de Oaxaca. 


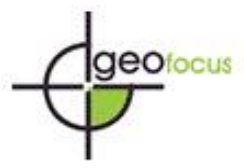

Suárez, M. E. (2020). Simulación del movimiento de partículas contaminantes sobre zonas de vulnerabilidad en el acuífero de los valles centrales de Oaxaca. GeoFocus, Revista Internacional de Ciencia y Tecnología de la Información Geográfica, 25, 3-25. http://dx.doi.org/10.21138/GF.653

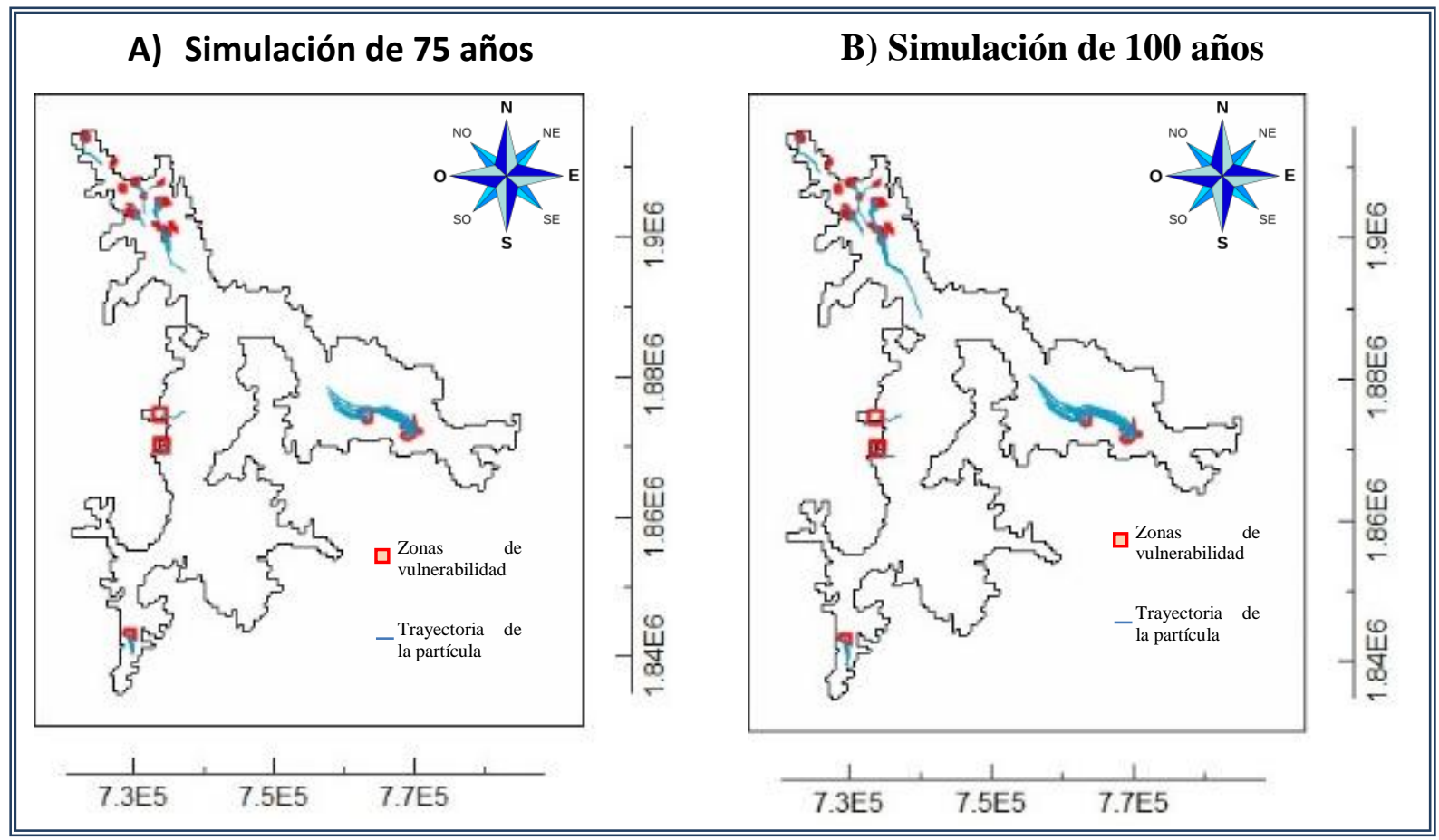

Figura 9. Simulaciones del movimiento de partículas a 75 y 100 años sobre las zonas de vulnerabilidad en el acuífero de los Valles Centrales de Oaxaca.

En la figura 10 se aprecia con mayor detalle el recorrido que hacen las partículas desde el origen en las zonas vulnerables hasta sus posiciones finales en un tiempo de 9942.6 años satisfaciendo algún criterio de término. El acuífero tiene una exageración vertical de 10 y se muestra en la figura con la vista superior, lateral, frontal y en tercera dimensión. Las longitudes de desplazamiento expresadas anteriormente son medidas desde la vista superficial en 2D, los desplazamientos completos de las partículas aumentan al tomar en cuenta su movimiento en tercera dimensión.

Las zonas donde las partículas se desplazaron con una mayor distancia y rapidez y que requieren una especial e inmediata protección son principalmente el área de cultivo localizada a $1.5 \mathrm{~km}$ al noroeste de San Lucas Quiavini, el cuerpo de agua localizado a $2.5 \mathrm{~km}$ al noroeste de San Miguel Tlanichico, los terrenos de cultivo entre Guadalupe y San Sebastián Etla y la zona urbana de San Francisco Telixtlahuaca. 


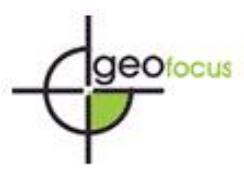

Suárez, M. E. (2020). Simulación del movimiento de partículas contaminantes sobre zonas de vulnerabilidad en el acuífero de los valles centrales de Oaxaca. GeoFocus, Revista Internacional de Ciencia y Tecnología de la Información Geográfica, 25, 3-25. http://dx.doi.org/10.21138/GF.653
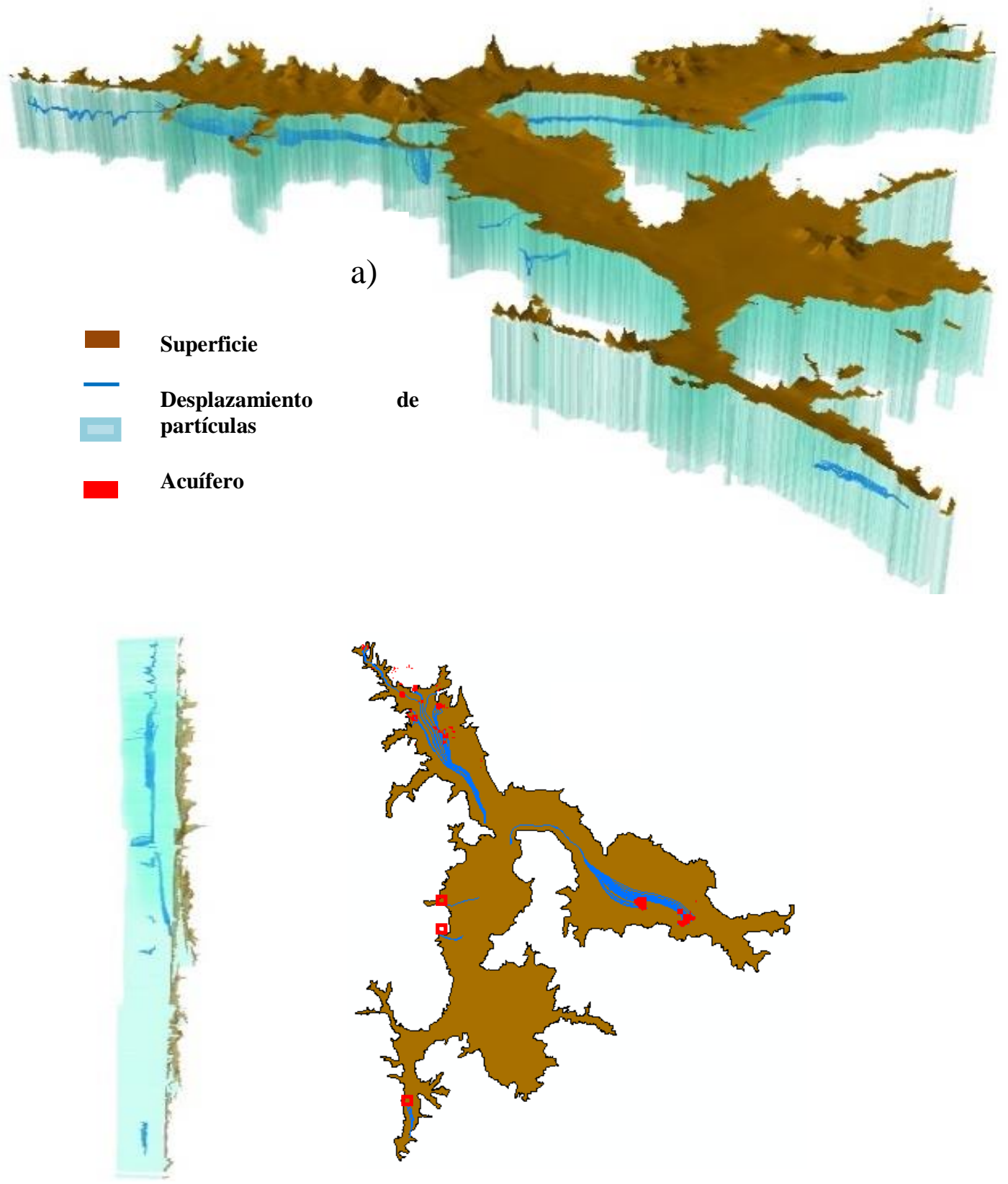

c)

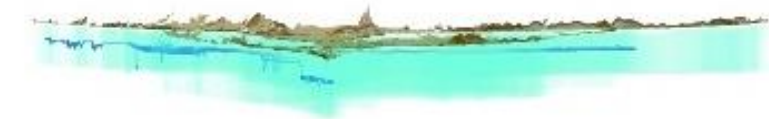

Figura 10. Simulación del movimiento de partículas en el acuífero de los Valles Centrales de Oaxaca desde las zonas vulnerables hasta su posición final. a) Vista en 3D, b) Vista lateral, c) Vista frontal, d) Vista superior 


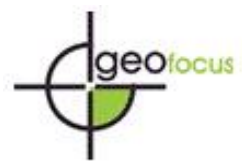

Suárez, M. E. (2020). Simulación del movimiento de partículas contaminantes sobre zonas de vulnerabilidad en el acuífero de los valles centrales de Oaxaca. GeoFocus, Revista Internacional de Ciencia y Tecnología de la Información Geográfica, 25, 3-25. http://dx.doi.org/10.21138/GF.653

\section{Discusión y valoración de hallazgos}

\subsection{Zonas potenciales de recarga con la metodología Matus}

Los cinco parámetros utilizados en las zonas potenciales de recarga pueden obtenerse de diferentes formas, especialmente el parámetro de cobertura vegetal permanente y el uso de suelo y vegetación. Para la cobertura vegetal permanente Donis (2015) plantea una sobreposición de los mapas de pendiente, textura de suelo y geología sobre el parámetro de tipo de suelo y vegetación para así conseguir el parámetro de cobertura permanente. Por otra parte, Cortés (2016) clasificó la vegetación con valores reportados por Meneses (2014) según el índice normalizado de la vegetación (NDVI) para las diferentes coberturas vegetales y ponderó de acuerdo al tipo de vegetación resultante. En este trabajo, se utilizó un estudio mundial de Mao-Ning y Jetz (2014) quien analizó la cubierta de la superficie de la Tierra a una escala de $1 \mathrm{~km}$. La ventaja de utilizar este estudio es que los resultados representados en formato ráster ya vienen en porcentaje de cobertura, tal como lo recomienda Matus (2007), no obstante, la resolución de $1 \mathrm{~km}$ hace que haya incertidumbre en los resultados.

El uso del suelo es un elemento que establece el grado de cómo una determinada actividad o cambio de uso, influye tanto en el deterioro de sus características como la erosión y la compactación, así como en la reducción de la infiltración y/o recarga hídrica (Matus, 2007). Abellán (2016) menciona que el proceso de urbanización es uno de los parámetros que más influye en la reducción de la infiltración y la eliminación de la vegetación natural. Esto se traduce en la interrupción de equilibrio de la hidrología superficial y la recarga de acuíferos. Sin embargo, a pesar de la gran importancia de las zonas urbanas en el estudio de las zonas de recarga, la metodología de Matus no lo toma en cuenta. Por lo anterior se justifica la modificación de la clasificación de uso de suelo ponderando en las zonas con más urbanización un valor de 1 .

Matus (2007) resalta que es necesario enfatizar en la cantidad de agua infiltrada en la zona de recarga, está en función de la cantidad de lluvia que cae y escurre hacia estas zonas y de las zonas potenciales de recarga. En este planteamiento, la precipitación es considerada como la fuente principal de la recarga, es decir, puede haber una gran cantidad de recarga en un lugar con precipitación alta y con potencial de recarga moderada, así mismo, puede no existir recarga alguna en una zona con baja o nula precipitación, aunque las zonas potenciales se muestren como muy alta.

\subsection{Zonas de vulnerabilidad a contaminantes con la metodología DRASTIC}

Se optó por evaluar el acuífero de los Valles Centrales de Oaxaca con la metodología de Aller et al. (1987) debido a que se tiene información de los siete parámetros que requiere DRASTIC en un lapso de tiempo desde 2001 hasta el 2009 para los parámetros que varían con el tiempo (recarga y profundidad del nivel freático). Resulta erróneo utilizar información de diferentes años o lapsos de tiempo, ya que estos dos parámetros dependen específicamente de la precipitación. Dávila et al. (2014) mencionan que, la evaluación DRASTIC es válida para situaciones en un tiempo específico, por esta razón, no se puede extrapolar la vulnerabilidad del acuífero a largo plazo.

Se encontró un promedio de nueve años de análisis de la profundidad del nivel estático (CONAGUA, 2013). En general, la variación obedece a la topografía, es decir, la profundidad del nivel estático aumenta conforme aumenta la altitud del terreno. Cabe mencionar que los niveles estáticos más 


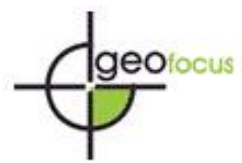

Revista Internacional de Ciencia y Tecnología de la Información Geográfica

International Review of Geographical Information Science and Technology

Suárez, M. E. (2020). Simulación del movimiento de partículas contaminantes sobre zonas de vulnerabilidad en el acuífero de los valles centrales de Oaxaca. GeoFocus, Revista Internacional de Ciencia y Tecnología de la Información Geográfica, 25, 3-25. http://dx.doi.org/10.21138/GF.653

profundos $(24 \mathrm{~m})$ se encuentran en áreas donde se han concentrado las extracciones de agua subterránea, específicamente al sur del acuífero, tomando valores de curvas de igual profundidad al nivel estático que varían desde los 14 hasta los 24 metros en el Valle de Zimatlán. En el Valle de Tlacolula se presentan otros halos de curvas de igual profundidad al nivel estático que varían de 4 a 14 metros, ocasionados también por la concentración del bombeo en esas áreas (UACh-Unidad Gestora de Servicios Tecnológicos, 2010). En lugares con poca profundidad da lugar a que exista un menor tiempo de viaje de un contaminante para alcanzar el nivel freático (Jiménez et al., 2000). En la zona vadosa o no saturada, Foster (1991) señala que una zona saturada de $10 \mathrm{~m}$ permite una completa autopurificación de efectos nocivos. Aller et al. (1987) además de tomar en cuenta el espesor de la zona saturada, también toman en cuenta el tipo de roca que la conforman. El acuífero de los Valles Centrales es de tipo libre, por lo tanto, no posee una roca impermeable que proteja al acuífero, lo anterior además de la escasa información, justifica el haber tomado la zona saturada y la litología del acuífero con la misma información cartográfica, pero con diferentes ponderaciones para cada parámetro.

El parámetro de recarga neta se puede calcular mediante la generación de polígonos de Thiessen (Coello y Galárraga, 2002), también restando a la precipitación total únicamente la evapotranspiración específica de cada zona (Becerril, 2013), o bien, realizando un balance de aguas subterráneas. Sin embargo, la recarga neta normalmente está sujeta a grandes errores e incertidumbre (Kumar y Seethapathi, 2002). Se probaron varios métodos para la evaluación de este parámetro, uno de ellos fue APLIS (por sus parámetros Altitud, Pendiente, Litología, Infiltración preferencial y Suelo) desarrollado por Andreo et al. (2014). Se obtuvo como resultado el porcentaje de precipitación que infiltra al acuífero, no obstante, por tratarse de una metodología para acuíferos carbonatados los resultados sobrepasaron los rangos de infiltración de un acuífero normal. El método que más se acercó a valores de estudios a escala local como Belmonte-Jiménez (2005) evaluando el valle de Zaachila y Martínez et al. (2014) para el valle de Ocotlán es la ecuación empírica de Kumar y Seethapathi (2002), aunque este método solo evalúa la precipitación y no la zona de infiltración. Belmonte-Jiménez (2005) generalizó la recarga neta con un promedio de 96 mm/anuales para el valle de Zaachila y Martínez et al. (2014) presentaron una recarga de $31 \mathrm{~mm} /$ anuales para Ocotlán. Los resultados obtenidos en este análisis con la ecuación de Kumar indican valores de 31 a $165 \mathrm{~mm} /$ anuales en toda la superficie de la subcuenca Alto Atoyac, presentando valores cercanos a 100 mm/anuales para el valle de Zaachila y de 35 a 70 mm/anuales para Ocotlán.

El conocimiento de la conductividad hidráulica $(\mathrm{K})$, tanto saturada como no saturada, es necesario como dato inicial en modelos para predecir el comportamiento del flujo del agua o fluidos a través del subsuelo (Rojas et al., 2008). Se utilizaron los análisis de la UACh-Unidad Gestora de Servicios Tecnológicos (2010) y de Bautista-Perdomo (2013) para obtener la conductividad hidráulica en toda la subcuenca, no obstante, se discriminaron los valores de la UACh en el aluvial ya que su análisis solo consta de la calibración de un modelo de flujo. Se encontraron valores muy altos de conductividad en la zona aluvial de la subcuenca de hasta 45 metros por día.

Rodríguez-Caro (2016) menciona que el suelo es el primer obstáculo físico que se encuentra todo contaminante en su camino de incorporación al acuífero. En dicha capa tienen lugar un conjunto de procesos que afectaran tanto a la cantidad como a las características de las sustancias que lo atraviesan, que dependerán de las características del suelo (textura, humedad, composición, etc.), por lo que su importancia a la hora de valorar la vulnerabilidad debe de considerarse como elevada. Aller et al. (1987) por su parte, le asigna a este parámetro el peso de 2, uno de los pesos más bajos en esta metodología, solo antes de la topografía. Rivera et al. (2005) menciona que la topografía tiene una gran influencia en el poder erosivo del agua de escorrentía en el suelo, en pendientes muy pronunciadas el grosor del suelo 


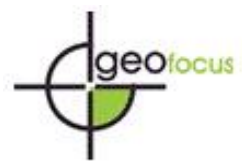

Suárez, M. E. (2020). Simulación del movimiento de partículas contaminantes sobre zonas de vulnerabilidad en el acuífero de los valles centrales de Oaxaca. GeoFocus, Revista Internacional de Ciencia y Tecnología de la Información Geográfica, 25, 3-25. http://dx.doi.org/10.21138/GF.653

disminuirá por efecto de la erosión, además, al ser mayor la escorrentía es menor la infiltración y la recarga al acuífero. Al ser un parámetro que influye tanto en la escorrentía, la erosión del suelo, la profundidad del nivel freático y la infiltración, la topografía debería de tener un valor de peso más alto en la metodología DRASTIC. Matus (2009) considera que la topografía es el parámetro que más afecta a la infiltración con un $27 \%$ en el peso total de los 5 parámetros. La superficie aluvial tiene una pendiente inferior a 10 por lo que la infiltración es mayor.

Aragón et al. (2012) indican que con la modificación de los rangos del método DRASTIC es posible acercarse a la realidad en la representación de las zonas más vulnerables a la contaminación, eliminando de manera importante la subjetividad del método. El proceso de calibración debe ser parte esencial en este tipo de estudios para cumplir con el propósito de los mapas de vulnerabilidad. A pesar de ello, Aller et al. (1987) afirman que los factores de peso de las variables deben mantenerse en cualquier estudio que se aplique, ya que la metodología fue aplicada en diversos escenarios hidrológicos y analizaron las distintas formas en que los parámetros pueden interactuar, por lo que mantener los valores reduce la subjetividad. Por ello se justifica el no cambiar los valores de peso de los parámetros DRASTIC, aun teniendo el análisis de la sensibilidad de cada variable.

Belmonte et al. (2003) considera el $91.76 \%$ del valle de Etla con vulnerabilidad alta y un $8.24 \%$ con vulnerabilidad moderada. Los resultados de este trabajo no concuerdan con los mencionados anteriormente, ya que para el valle de Etla las zonas que predominaron fueron las de vulnerabilidad moderada y baja y solo en la parte del aluvial se encontró vulnerabilidad alta. El resultado contrasta por la delimitación del valle que pudo a ver sido específicamente en el aluvial ya que Belmonte et al. (2003) solo tomó en cuenta $290 \mathrm{~km}^{2}$ del valle, menos de la tercera parte.

Martínez et al. (2014) encontraron en el valle de Ocotlán que la vulnerabilidad alta ocupa casi el $100 \%$ de la zona. Este resultado contrasta mucho con los obtenidos en esta tesis, encontró que poco menos del $50 \%$ del valle de Ocotlán tiene una vulnerabilidad baja y menos del $15 \%$ es altamente vulnerable. Es posible que los resultados varíen por la escala de la realización del estudio y también por la información utilizada para obtener cada uno de los parámetros.

\subsection{Modelación y simulación}

Los acuíferos son tan complejos, que no es posible estudiarlos a detalle (Rushton, 2003). Anderson y Woessner (1992) señalan que los modelos empleados en estudios de acuíferos solo serán confiables si los datos utilizados así lo son, es decir, depende de su cantidad y calidad. No obstante, hay parámetros que tienen una gran incertidumbre ya que los diferentes métodos utilizados para su obtención, utilizan información que se interpola o se generaliza como en el caso de la recarga del acuífero y la conductividad hidráulica.

Bautista-Perdomo (2013) determinó el modelo de flujo del sistema acuífero Alto Atoyac y se operó ante diferentes escenarios para conocer la relación de extracción y niveles piezométricos, por lo que fue necesario la calibración. Del mismo modo, UACh-Unidad Gestora de Servicios Tecnológicos (2010) realizó el modelo de flujo para la predicción y la propuesta de alternativas para su manejo por lo que el tipo es de predicción. En el presente trabajo, la modelación del acuífero se utilizó para obtener el flujo del sistema y se empleó en la simulación de partículas contaminantes, por lo que la aplicación fue genérica y no se requirió de alguna calibración. El modelo no es adecuado para la predicción ni la evaluación del comportamiento del nivel freático a largo plazo. 


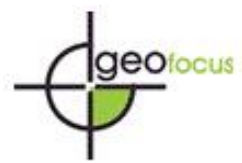

Suárez, M. E. (2020). Simulación del movimiento de partículas contaminantes sobre zonas de vulnerabilidad en el acuífero de los valles centrales de Oaxaca. GeoFocus, Revista Internacional de Ciencia y Tecnología de la Información Geográfica, 25, 3-25. http://dx.doi.org/10.21138/GF.653

Frind et al. (2006) mencionan que 50 años (18250 días) son suficientes para que se atenúen los contaminantes más persistentes en un acuífero. Sin embargo, Pollock (2016) recalca que MODPATH no considera ningún parámetro de degradación o atenuación de contaminantes, solo el fenómeno de advección, por ello, la simulación de contaminantes máxima se alcanzó a los 9942.6 años hasta que llegó al límite del modelo, a un sumidero o fuente interna.

El modelo ha sido construido para simular advectivamente sólo el transporte. Otros procesos que influyen en el transporte de contaminantes y que no son tomados en cuenta por MODPATH son la difusión, adsorción, dispersión hidrodinámica y mecánica, disolución de solutos, mineralización del suelo, reacción química, desintegración radiactiva, degradación biológica de contaminantes orgánicos y procesos de reducción similares, como la desnitrificación. Los tiempos de viaje están asociados al contaminante. El transporte que está significativamente influenciado por cualquiera de los procesos anteriores requiere el uso de más modelos sofisticados como MT3DMS de MODFLOW (Zheng y Wang, 1999).

Montoya (2016) realizó un análisis de partículas en una presa de relaves con MODPATH modelando con conductividades hidráulicas inferiores a 5 E-7 m/s. En poco más de 40 años las partículas recorrieron más de 1 kilómetro hasta llegar a un dren. En otro estudio Ríos et al. (2007) desarrollaron una simulación de partículas en 20 años en Valle del Cauca Colombia. Las partículas se desplazaron más de 10 kilómetros en este tiempo, algunos llegando a los drenes cercanos. Las conductividades en el acuífero de los Valles Centrales de Oaxaca, que se obtuvieron oscilan de los 0.05 a los 45 metros/día o de 5.787 E7 a 5.2083 E-4 metros/segundo. Estos datos concuerdan con las velocidades de desplazamiento registrado, por ejemplo; al norte de la Villa de Etla con 4.20E-5 metros/segundo o en San Francisco Telixtlahuaca con 9.5E-6 metros /segundo. El acuífero de Oaxaca contrasta en sus velocidades de simulación con la evaluación de Montoya (2016) y Rios et al. (2007), esto se debe al tipo de acuífero, la velocidad de los flujos y sus propiedades como porosidad y conductividad hidráulica.

5.4. Enfoque combinado de recarga potencial, vulnerabilidad, transporte advectivo y simulación de partículas

El método de vulnerabilidad DRASTIC tiene naturaleza intrínseca. Algunos estudios con este método muestran exitosos resultados, sin embargo, otros casos muestran una clara discrepancia entre los mapas de vulnerabilidad y las concentraciones de contaminantes observados en las aguas subterráneas (Tavares-Riveiro, 2015). Los estudios de campo muestran que los métodos que incorporan información sobre el tipo de cobertura de la tierra y/o el tipo de actividades asociadas a humanos (por ejemplo, el tipo y la naturaleza de las prácticas agrícolas) y no sólo las características intrínsecas, se desempeñan mejor que los métodos puramente intrínsecos (Stigter et al., 2006). Por ello, los parámetros de uso de suelo y cobertura vegetal permanente presentes en la metodología de Matus (2009), son un complemento idóneo para eliminar las desventajas que plantea Stigter de depender únicamente de las características intrínsecas del acuífero.

El enfoque combinado basado en un método de vulnerabilidad, de transporte advectivo y de simulación de partículas, permite comprender mejor la susceptibilidad a la contaminación en un acuífero (Gárfias et al., 2017). Aunado a esto, la intersección de las zonas de mayor vulnerabilidad junto con las zonas de mayor recarga o infiltración propone un mayor grado de susceptibilidad en dichas zonas. Ante la imposibilidad del DRASTIC de considerar el sistema de flujo del acuífero ya que son únicamente calculados para una determinada posición y no reflejan las condiciones hidrodinámicas del flujo del agua 


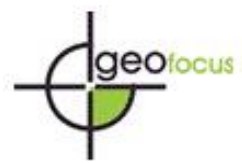

Suárez, M. E. (2020). Simulación del movimiento de partículas contaminantes sobre zonas de vulnerabilidad en el acuífero de los valles centrales de Oaxaca. GeoFocus, Revista Internacional de Ciencia y Tecnología de la Información Geográfica, 25, 3-25. http://dx.doi.org/10.21138/GF.653

subterránea, la combinación con un modelo de flujo y simulación de partículas permitió identificar zonas más vulnerables que requieren especial protección (figuras 32, 33, 34 y 35).

Gárfias et al. (2017) combinaron las zonas vulnerables junto con las zonas de desplazamiento subterráneo de las partículas para crear zonas de vulnerabilidad más elevadas. En su estudio, las partículas se simularon hacia atrás en el tiempo, las zonas finales fueron los pozos para encontrar los puntos que podrían contaminarlos. Las partículas en este estudio comenzaron en las zonas vulnerables y se identificaron las zonas hacia donde se desplazaban y cuales se desplazaban más rápido.

\section{Conclusiones}

Los mapas de vulnerabilidad DRASTIC y la combinación con zonas potenciales de recarga, además de servir para la elaboración de políticas para la protección del acuífero de los Valles Centrales de Oaxaca, podrían constituir una herramienta para la gestión del territorio y conducir a evaluaciones de vulnerabilidad más concluyentes sobre el uso del territorio y a la toma de medidas de protección contra la contaminación. El uso combinado de las zonas de vulnerabilidad, de potencial de recarga y de transporte advectivo, permitió identificar zonas muy específicas que requieren de especial protección. En cualquiera de esas áreas, actividades potencialmente contaminantes podrían provocar el deterioro de la calidad del agua subterránea.

En la simulación no todas las partículas se movieron uniformemente con el tiempo, los desplazamientos dependieron de factores como la conductividad hidráulica del estrato de roca que atravesaban y de las velocidades de flujo. Las zonas más vulnerables y con mayor desplazamiento advectivo no solo serían un peligro para la calidad de agua en el sitio, si no que podrían afectar a pozos o comunidades a kilómetros de distancia. Entre las zonas identificadas están los terrenos de cultivo de San Lucas Quiavini, Tlacolula, el cuerpo de agua perteneciente a San Miguel Tlanichico, Zaachila, la zona agrícola entre San Sebastián y Guadalupe Etla y la zona urbana en San Francisco Telixtlahuaca.

Mediante el modelo de vulnerabilidad presentado en este trabajo, no es posible afirmar que es el mejor método para la zona de estudio. Es necesario validar los resultados mediante mediciones de la contaminación en campo. Sin embargo, los resultados permiten vislumbrar aquellas zonas potencialmente vulnerables a la contaminación dentro de la región de estudio. Así, este tipo de estudios constituyen el punto de partida para nuevas investigaciones que permitan determinar y seleccionar la mejor alternativa de evaluación, al igual que sirve de guía para la realización de monitoreos de la calidad del agua del acuífero.

\section{Referencias bibliográficas}

Abellan, A. (2016): Impactos de la urbanización en el ciclo del agua. Iagua. [Consulta: 16-abril-2018]. Disponible en https://www.iagua.es/blogs/ana-abellan/impactos-urbanizacion-ciclo-agua.

Albinet, M. y Margat J. (1970) “Cartographie de la vulnérabilité à la pollution des nappesd'eausouterraine" Bull. BRGM, 2 ème serie, 3,4, 13-22 p.

Aller, L., Bennet, T., Lher, J. y Hackett, G. (1987): “DRASTIC: A standardized system for evaluating groun water pollution potencial using hydrogeologic setting. Unite States Enviromental Protectuin Agency". Chicago, Ed. AcademicPress. 


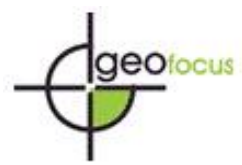

Suárez, M. E. (2020). Simulación del movimiento de partículas contaminantes sobre zonas de vulnerabilidad en el acuífero de los valles centrales de Oaxaca. GeoFocus, Revista Internacional de Ciencia y Tecnología de la Información Geográfica, 25, 3-25. http://dx.doi.org/10.21138/GF.653

Anderson, P. y Woessner, W. (1992): "Applied Groundwater Modeling Simulation of Flow and Advective Transport”. San Diego, Ed. AcademicPress.

Andreo, B., Vías, J., López-Geta, J.A., Carrasco, F., Durán, J.J., Jiménez, P. (2004): "Propuesta metodológica para la estimación de la recarga en acuíferos carbonáticos ", Boletín Geológico y Minero, 115,2 .

Aragón, M. D., Navarro Mendoza, S., Belmonte Jiménez, S. y Torres, G. (2012) "Calibración del método DRASTIC en acuíferos utilizando nitratos como indicador”, Ingeniería Hidráulica y Ambiental, 33,2, pp. 21-30.

Bautista-Perdomo, J.M. (2013): Modelo de flujo del sistema acuífero de la cuenca Alto Atoyac, Oaxaca. Tesis de Maestría. Centro Interdisciplinario de Investigación para el Desarrollo Integral Regional Unidad Oaxaca, IPN.

Becerril, M.A. (2013): "Vulnerabilidad natural del agua subterránea en el valle de Tula, Hidalgo". Tesis de Maestría. Universidad Nacional Autónoma de México, D.F. México. 15-44 p.

Bokova, I. (2010): “Día mundial del agua”. UNESCO. Elementalwatson, 1, pp. 5-12.

Cavero, M. (2013): MODPATH versión 6. Colombia, Gidahatari. [Consulta: 20-02-2018]. Disponible en http://gidahatari.com/ih-es/modpath-version-6.

Coello, X. y Galárraga, H. (2002): Metodologías para el análisis de la vulnerabilidad de acuíferos en medios urbanos, el caso de Quito, Ecuador, Quito, Groundwater and Human Development.

CONAGUA (Comisión Nacional del Agua) (2013): Integración del estudio técnico justificativo de los acuíferos: Ostuta, Río Verde-Ejutla y Valles Centrales, en el estado de Oaxaca, Oaxaca, Diario oficial de la federación.

Cortés, M. (2016): Potencial de recarga hídrica y morfometría de la microcuenca Rio Grande, Oaxaca y su relación con unidades geográficas, administrativas y de manejo forestal. Tesis de Maestría. División de estudios de postgrado, Universidad de la Sierra Juárez, Oaxaca.

Custodio, G. (1998): "Recarga a los acuíferos: aspectos generales sobre el proceso, la evolución y la incertidumbre", Boletín Geológico y Minero, 109,4, pp. 13-29.

Donis, L., F. (2015): Identificación de zonas de recarga hídrica en la microcuenca del río negro. Tesis de licenciatura. Universidad Rafael Landíver. Facultad de ciencias ambientales y agrícolas. Guatemala.

Esteller, M.V., Quentin, E., y Díaz-Delgado, C. (2002): “Uso de sistemas de información geográfica (SIG) para la determinación de parámetros utilizados en la construcción de mapas de vulnerabilidad de acuíferos", Revista Latino-Americana de Hidrogeología, 2,1, pp.17-30.

Foster, S. e Hirata, R. (1991): Determinación del Riesgo de contaminación de aguas subterráneas, Centro Panamericano de Ingeniería Sanitaria y Ciencias del Ambiente (CEPIS). Lima, Hidrogeología ambiental.

Gárfias, J., Llanos, H., Franco, R. y Martel, R. (2017): "Estimación de la vulnerabilidad del acuífero del valle de Toluca mediante la combinación de un método paramétrico y el transporte advectivo", Boletín Geológico y Minero. México, 128, pp. 25-42.

Gogu, R.C y Dessargues, A. (2000): "Currenttrends and future challenges in groundwater vulnerability assessment using overlay and index methods", Environmental Geology, 39,6, pp. 549-559. 


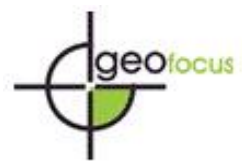

Suárez, M. E. (2020). Simulación del movimiento de partículas contaminantes sobre zonas de vulnerabilidad en el acuífero de los valles centrales de Oaxaca. GeoFocus, Revista Internacional de Ciencia y Tecnología de la Información Geográfica, 25, 3-25. http://dx.doi.org/10.21138/GF.653

Harbaugh, A.W. (2005): MODFLOW-2005. The U.S. Geological Survey Modular Ground-Water Model-the Ground-Water Flow Process. U.S. Department of the Interior U.S. Geological Survey, Reston, Virginia.

INEGI (Instituto Nacional de Estadística, Geografía e Informática). 2001. Continuo de Elevaciones Mexicano 3.0 (CEM 3.0). Resolución a 15 m.

INEGI (Instituto Nacional de Estadística, Geografía e Informática). 2005. Conjunto de datos vectoriales geológicos. Continuo nacional. Escala 1:1 000000.

INEGI (Instituto Nacional de Estadística, Geografía e Informática). 2015. Conjunto de datos vectoriales de uso de suelo y vegetación. Escala 1:250 000, serie V.

INEGI (Instituto Nacional de Estadística, Geografía e Informática). 2016. Cartografía estadística urbana y rural amanzanada. Oaxaca. Escala 1:250 000.

INIFAP (Instituto Nacional de Investigación Forestales y Agropecuarias) y CONABIO (Comisión Nacional para el Conocimiento y uso de la Biodiversidad). 2001. Edafología, escala 1:1000000.

Jiménez, B. S. I., Bautista Belmonte, A., Navarro Mendoza, S. M., y Aragón Sulik, M. D. (2000): "Determinación de la vulnerabilidad de acuíferos con los métodos AVI, GOD y DRASTIC", In Congreso Interamericano de Ingeniería Sanitaria y Ambiental, México 27, pp. 1-4.

Koohestani, N., Halaghi M. y Dehghani, A. (2013): "Numerical simulation of groundwater level using MODFLOW software (A case study: Narmabwatershed, Golestanprovince)", International Journal of Advanced Biological and Biomedical Research, Reston, Virginia. 1,8, pp. 858-873.

Kumar, C. P., y Seethapathi, P.V. (2002): “Assessment of natural groundwater recharge in Upper Ganga Canal commandarea", Journal of Applied Hydrology, 15,4, pp. 13-20.

Mao-Ning, T. y Jetz, W. (2014): “A global 1-km consensus land-cover product for biodiversity and ecosystem modelling”. Global Ecology and Biogeography, 23,9, pp. 1031-1045.

Martínez, A., García, L.A., Belmonte-Jiménez, S. y De Guevara, L.A. (2014): Determinación de la vulnerabilidad intrínseca con DRASTIC del acuífero del Valle de Ocotlán, Oaxaca. Centro Interdisciplinario de Investigación para el Desarrollo Integral Regional Unidad Oaxaca.

Martínez, I. R. y Odvin E. (2017): Evaluación de la vulnerabilidad del acuífero del valle de sébaco ante la contaminación y sobrexplotación y propuesta de un plan de gestión del recurso hídrico. Tesis de maestría. Escuela Regional de Ingeniería Sanitaria y Recursos Hidráulicos (ERIS). Universidad de San Carlos de Guatemala.

Matus Silva, O. D. (2007): Elaboración participativa de una metodología para la identificación de zonas potenciales de recarga hídrica en subcuencas hidrográficas, aplicada a la subcuenca del río Jucuapa, Matagalpa. Tesis de Maestría. CATIE, Turrialba, Costa Rica.

Matus, O., Faustino, J. y Jimenez, F. (2009): Guía para la identificación participativa de zonas con potencial de recarga hídrica: Aplicación práctica en la subcuenca del rio Jucuapa, Nicaragua. Centro Agronómico Tropical de Investigación y Enseñanza. Turrialba, Costa Rica. 


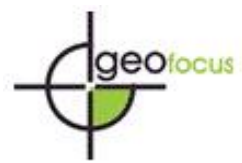

Suárez, M. E. (2020). Simulación del movimiento de partículas contaminantes sobre zonas de vulnerabilidad en el acuífero de los valles centrales de Oaxaca. GeoFocus, Revista Internacional de Ciencia y Tecnología de la Información Geográfica, 25, 3-25. http://dx.doi.org/10.21138/GF.653

Meneses, C., L. (2009): Análisis del Índice Normalizado de la Vegetación (NDVI) para la detección de degradación de la cubierta forestal en México 2008-2009. Italia, Organización de las Naciones Unidas para la Agricultura y la Alimentación, Departamento Forestal, Roma.

Montoya, S. (2016): Tutorial del Cálculo de Tiempo de Transporte de Trazador con MODFLOWMODPATH. [Consulta: 12-enero-2018]. https://www.youtube.com/watch?v=j57FwiS98GI\&t=816s

Pollock, D. W. (2016): User Guide for MODPATH Version 6A Particle-Tracking Modelfor MODFLOW. U.S. Reston, Virginia, Department of the Interior U.S. Geological Survey.

Pollock, D.W. (2012): User Guide for MODPATH Version 6A Particle-Tracking Model for MODFLOW. Reston, Virginia, U.S. Department of the Interior. U.S. Geological Survey.

Ríos, L. y OtálvaroVélez, M.V. (2007): "Estimación de la vulnerabilidad de acuíferos mediante un modelo cuantitativo-Hydrus 1D", Avances en recursos hidráulicos, 15, pp. 23-34.

Rivera, F., Gutiérrez, A., Val, R., Mejía, R., Sánchez, P. A. y Aparicio, J. (2005): La medición de sedimentos en México. México, IMTA.

Rodríguez-Caro, J. B., Rubio Campos, J. C., Luque, J. A. (2003): Las aguas minerales, mineromedicinales y termales de la provincia de Jaén, Instituto Geológico y Minero de España, IGME.

Rojas, L. M., Rojas-Palacio, H., Menjívar-Flórez, J. C. (2008): “Estimación de la conductividad hidráulica saturada in situ en un suelo tratado con vinaza". Acta Agron (Palmira), 57 ,2, pp. 125-128.

Rushton, K. R. (2003): Groundwater hidrology conceptual and computacional models. England, Ed. John Wiley y Sons.

Stigter, T. Y., Ribeiro, L., Carvalho Dill, A.M.M. (2006): "Evaluation of anintrinsic and a specific vulnerability assessment method in comparison with ground water salinisation and nitrate contamination levels in two agricultural regions in the south of Portugal", Hydrogeology Journal, 14,1- 2, pp. 79-99.

Tavares-Ribeiro, L. F. (2015) Vulnerabilidad de acuíferos. Investigador Prometeo, Escuela Superior Politécnica del Litoral, Guayaquil, Ecuador.

Vargas, M.C. y Tosse O.D. (2010): Propuesta metodológica para la evaluación de la vulnerabilidad intrínseca de los acuíferos a la contaminación. Ministerio de Ambiente, Vivienda y Desarrollo Territorial. Dirección de Ecosistemas - Grupo de Recurso Hídrico. República de Colombia.

UACh-Unidad Gestora de Servicios Tecnológicos. (2010): Plan de manejo acuífero Valles Centrales, en el estado de Oaxaca.

Velásquez, L.I. (2017): Metales cancerígenos contiene el agua en Oaxaca. Oaxaca, NVINoticias. [Consulta: 09-01-2018]. Disponible en https://www.nvinoticias.com/nota/54409/metales-cancerigenoscontiene-el-agua-en-oaxaca.

Zheng, C., Wang, P.P., (1999): MT3DMS: A modular three-dimensional multispecies transport model for simulation of advection, dispersion, and chemical reactions of contaminants in groundwater systems. Vicksburg,

MS. 
